\title{
Stabilization of charged and neutral colloids in salty mixtures
}

\author{
Sela Samin and Yoav Tsor: $*$ \\ Department of Chemical Engineering and The Ilse Katz Institute for Nanoscale Science and Technology, \\ Ben-Gurion University of the Negev, 84105 Beer-Sheva, Israel.
}

(Dated: September 14, 2018)

\begin{abstract}
We present a mechanism for the stabilization of colloids in liquid mixtures without use of surfactants or polymers. When a suitable salt is added to a solvent mixture, the coupling of the colloid's surface chemistry and the preferential solvation of ions leads to a repulsive force between colloids that can overcome van der Waals attraction. This repulsive force is substantial in a large range of temperatures, mixture composition and salt concentrations. The increased repulsion due to addition of salt occurs even for charged colloids. This mechanism may be useful in experimental situations where steric stabilization with surfactants or polymers is undesired.
\end{abstract}

The stability of colloidal dispersions is essential in material science and technology. Steric stabilization of colloids against the attractive van der Waals (vdW) forces can be achieved using surfactant or polymer molecules that are physically or chemically attached to the colloid's surface. Charged colloids can also be stabilized via the screened Coulomb repulsion, whose range depends on the Debye length $\kappa^{-1}$. In the celebrated Derjaguin, Landau, Verwey, and Overbeek (DLVO) theory, addition of salt to the suspension decreases the Debye length and the electrostatic repulsion leading eventually to coagulation and sedimentation of the colloids [1].

In recent years we began to better understand the differences between the electrostatics of pure solvents compared to liquid mixtures. The preferential wetting of one liquid component at the colloid surface [2, 3] affects the density of the ions, the electrostatics of the mixture, and the interaction between the colloids [4-12]. A key parameter is the selective solvation of the ions in the liquids [13-22].

In this paper we present a new method for the stabilization of electrically charged or neutral particles in solvent mixtures by addition of salt, without use of surfactants or polymers. The stabilization relies on (i) selective adsorption of one solvent on the colloids and (ii) a difference in the preferential solubilities of the anion and the cation in the solvents, namely a difference between the Gibbs transfer energy of moving the anion from one solvent to the other and the analogous Gibbs energy of the cation. These energies are often larger than the thermal energy [23, 24] and therefore particles can be stabilized even without other additives. In fact, requirements (i) and (ii) above are generally met because any surface is hydrophilic or hydrophobic to some extent and no two ions have identical solvation energies [23, 24]. The coupling of the colloid's surface chemistry and the preferential solvation of ions leads to a repulsive force between colloids that can overcome the van der Waals attraction. This repulsive force is large in a unexpectedly wide range of temperatures, mixture compositions and salt concentrations. This method preserves the chemical properties of

\footnotetext{
* tsori@bgu.ac.il
}

the surface and therefore is advantageous in cases where surfactants, polymers, or a chemical modification of the particle are undesired.

We consider relatively dilute mixtures in which ion-ion correlations effects are small [25, 26]. In order to isolate the effect of preferential solvation we ignore specific ionsurface interactions [27, 28]. These short range interactions can either enhance or counteract the stabilization mechanism we discuss and their magnitude is of specific nature.

We focus on a system composed of a binary aqueous mixture containing a $1: 1$ monovalent salt and confined between two identical flat plates. The plates are located at $z= \pm D / 2$ and their area is $S$. The mixture composition is given by the water volume fraction $\phi(0 \leq \phi \leq 1)$ while the cosolvent composition is given by $1-\phi$. The number densities of the point-like positive and negative ions are denoted by $n^{ \pm}$. The fluid between the plates is in contact with an electroneutral matter reservoir at composition $\phi_{0}$ and a salt concentration $n_{0}$.

The mean-field free energy density of the system is given by [17]:

$$
\begin{aligned}
f & =k_{B} T\left[f_{m}(\phi)+\frac{1}{2} C|\nabla \phi|^{2}\right]-\frac{1}{2} \varepsilon_{0} \varepsilon(\phi)(\nabla \psi)^{2} \\
& +e\left(n^{+}-n^{-}\right) \psi+k_{B} T \sum_{i= \pm} n^{i}\left[\left(\log \left(v_{0} n^{i}\right)-1\right)-\Delta u^{i} \phi\right],
\end{aligned}
$$

where $k_{B}$ is the Boltzmann constant, $T$ is the temperature, $\varepsilon_{0}$ is the vacuum permittivity, and $v_{0}=a^{3}$ is the molecular volume. $v_{0} f_{m}$ is the dimensionless mixing free energy density: $v_{0} f_{m}=\phi \log (\phi)+(1-\phi) \log (1-\phi)+$ $\chi \phi(1-\phi)$, where the Flory parameter is $\chi \sim 1 / T$. The mixture demixes for $T<T_{c}\left(\chi>\chi_{c}=2\right)$. The energetic cost of composition inhomogeneities is accounted for by the square-gradient term, where $C$ is a positive constant with units of inverse length. In the electrostatic energy in Eq. (1), $\psi$ is the electric potential and $\varepsilon$ is the dielectric constant, assumed to depend linearly on composition by $\varepsilon(\phi)=\varepsilon_{c}+\left(\varepsilon_{w}-\varepsilon_{c}\right) \phi$, where $\varepsilon_{w}$ and $\varepsilon_{c}$ are the water and cosolvent dielectric constants, respectively. The first term on the second line of Eq. (11) is the ions' electrostatic energy, where $e$ is the elementary charge. The 
first term in the sum is the ideal-gas entropy of the ions and the second term is the ion solvation energy. In our simple theory, the solvation energy is proportional to the local solvent composition and its strength is measured by the parameters $\Delta u^{i}[13,14]$. Here we are interested in salts where the asymmetry in the cation and anion solvation parameters, defined as $v \equiv\left(\Delta u^{+}-\Delta u^{-}\right) / 2$, is large. This is commonly satisfied in antagonistic salts where one ion is hydrophilic and the other is hydrophobic: $\Delta u^{+} \Delta u^{-}<0$.

The short range and electrostatic interactions between the fluid and the solid surfaces are given by the surface free energy density $f_{s}$ :

$$
f_{s}=k_{B} T \Delta \gamma \phi\left(\mathbf{r}_{s}\right)+e \sigma \psi\left(\mathbf{r}_{s}\right)
$$

where $\mathbf{r}_{s}$ is a vector on the colloid surface and $e \sigma$ is the surface charge density of the plates. The surface wettability is given by the parameter $\Delta \gamma$ that measures the difference between the solid-water and solid-cosolvent surface tensions.

The equilibrium state of the system is found by extremization of the grand potential

$\Omega=\int\left[f-k_{B} T\left(\lambda_{0}^{+} n^{+}+\lambda_{0}^{-} n^{-}+\mu_{0} \phi\right)\right] \mathrm{d} \mathbf{r}+\int f_{s} \mathrm{~d} \mathbf{r}_{s}$

where $\lambda_{0}^{ \pm}$and $\mu_{0}$ are the chemical potentials imposed by the species in the reservoir. This leads to the first Euler-Lagrange (EL) equation $\delta \Omega / \delta \phi=0$ :

$$
-C \nabla^{2} \phi+\frac{\partial f_{m}}{\partial \phi}-\sum_{i= \pm} \Delta u^{i} n^{i}-\varepsilon_{0} \frac{d \varepsilon / d \phi}{2 k_{B} T}(\nabla \psi)^{2}=\mu_{0}
$$

with the boundary condition $\mathbf{n} \cdot \nabla \phi=\Delta \gamma / C$, where $\mathbf{n}$ is a unit vector perpendicular to the surface. The EL equation for the potential $\delta \Omega / \delta \psi=0$ naturally yields Gauss' law: $-\nabla \cdot\left(\varepsilon_{0} \varepsilon(\phi) \nabla \psi\right)=e\left(n^{+}-n^{-}\right)$with the boundary condition $-\mathbf{n} \cdot \nabla \psi=e \sigma / \varepsilon_{0} \varepsilon(\phi)$. The densities $n^{ \pm}$from $\delta \Omega / \delta n^{ \pm}=0$ obey the Boltzmann distribution $n^{ \pm}=v_{0}^{-1} \exp \left(\mp \Psi+\Delta u^{ \pm} \phi+\lambda_{0}^{ \pm}\right)$, where $\Psi=e \psi / k_{B} T$ is the dimensionless potential.

A solution of the EL equations in a planar geometry yields the one dimensional profiles $\phi(z), \psi(z)$ and $n^{ \pm}(z)$. In Fig. 1]we plot the resulting profiles for a salty mixture between two electrically neutral and hydrophobic plates with a wettability $\Delta \gamma=0.2 / a^{2}$. The mixture has a bulk critical composition, $\phi_{0}=1 / 2$, and its temperature is far above $T_{c}, \Delta T=T-T_{c}=21.5 \mathrm{~K}$. The bulk concentration of the antagonistic salt is $n_{0}=20 \mathrm{mM}$ and the solvation parameters are $\Delta u^{+}=-\Delta u^{-}=v=6$.

The ionic profiles in Fig. 1 (b) show that an electrostatic diffuse layer (EDL) is realized near the plates, despite their electric neutrality. The reason is the adsorption of the cosolvent on the hydrophobic plates shown by the profile of $\phi$ in Fig. 11 (a). The cosolvent "drags" the hydrophobic anions and repels the hydrophilic cations. Hence, a net charge density develops in the vicinity of the

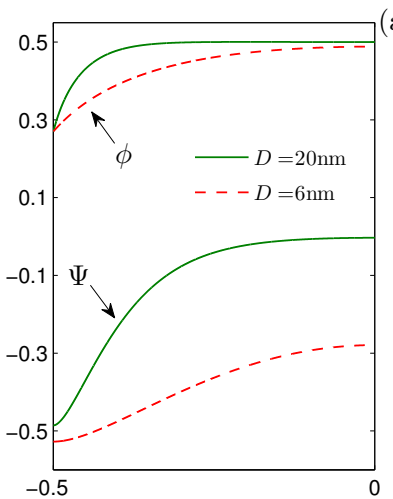

inin

$z / D$

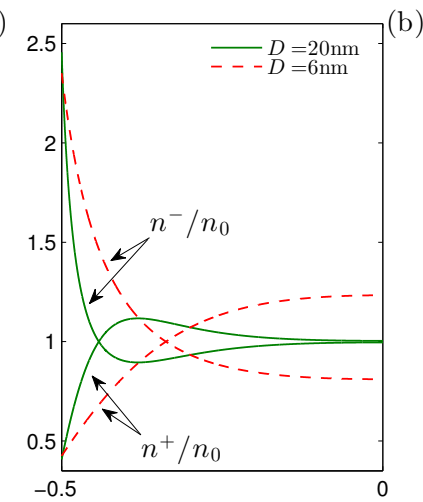

$z / D$

FIG. 1. Profiles of the (a) composition, dimensionless potential and (b) scaled ion densities between two electrically neutral plates immersed in a critical mixture $\left(\phi_{0}=1 / 2\right)$ at a temperature $\Delta T=T-T_{c}=21.5 \mathrm{~K}$ and containing $20 \mathrm{mM}$ of antagonistic salt $\left(\Delta u^{+}=-\Delta u^{-}=v=6\right)$. Here and in other figures the plates are hydrophobic with $\Delta \gamma=0.2 / \mathrm{a}^{2}$, corresponding to about $7 \mathrm{mN} / \mathrm{m}$. As an approximation to water-2,6-lutidine mixtures we used $T_{c}=307.2 \mathrm{~K}, v_{0}=39 \AA^{3}$, $C=\chi / a$ [29], $\varepsilon_{\text {lutidine }}=6.9$ and $\varepsilon_{\text {water }}=79.5$.

plates, giving rise to the electric potential profile shown in Fig. 1] (a).

The width of the adsorbed fluid layer is comparable to the bulk correlation length $\xi$. Beyond this distance $\phi(z)$ decays to its bulk value. However, electroneutrality dictates that the ionic profiles must compensate for the deviation from the bulk values near the plate. At surface separation of $D=20 \mathrm{~nm}$ (Fig. 11 (b), solid curves), this means that the anion (cation) concentration becomes smaller (larger) than the bulk value $n_{0}$ before it decays to $n_{0}$ at the midplane $(z=0)$, leading to a minimum (maximum) in the profile. On the other hand, at a distance of $D=6 \mathrm{~nm}$, the extremum is missing since the EDLs from each plate overlap, such that $n^{ \pm}(z=0) \neq n_{0}$. Such an overlap implies a repulsive osmotic force between the plates.

Using the profiles we calculate the osmotic pressure $\Pi$ between the plates at a given distance $D$ from $\Pi(D)=$ $P_{z z}-P_{0}$, where $P_{z z}=\phi \delta f / \delta \phi+n^{+} \delta f / \delta n^{+}+n^{-} \delta f / \delta n^{-}-$ $f-\varepsilon_{0} \varepsilon(\partial \psi / \partial z)^{2}$ is the $z z$ component of the Maxwell pressure tensor [17] and $P_{0}=P_{z z}\left(\phi_{0}, n_{0}^{ \pm}, \psi=0\right)$ is the bulk pressure. The interaction potential $U(D)$ between the plates is obtained from the osmotic pressure via $U(D)=-S \int_{\infty}^{D} \Pi\left(D^{\prime}\right) \mathrm{dD}^{\prime}$.

The interaction potential for the parameters of Fig. 1 with solvation asymmetry $v=6$ is plotted in Fig. 2 (red curve). At close separations the potential is attractive due to adsorption of the cosolvent on the plates, but a repulsive barrier of $\approx 10 k_{B} T$ appears in $U(D)$ at a distance denoted by $D_{\max }$ of a few nanometers. The barrier height $U_{\max }=U\left(D_{\max }\right)$ strongly depends on $v$. For an antagonistic salt with $v=4$ (blue curve) the barrier is much smaller, while for a hydrophilic salt with $v=0$ (green curve), the potential is purely attractive. 


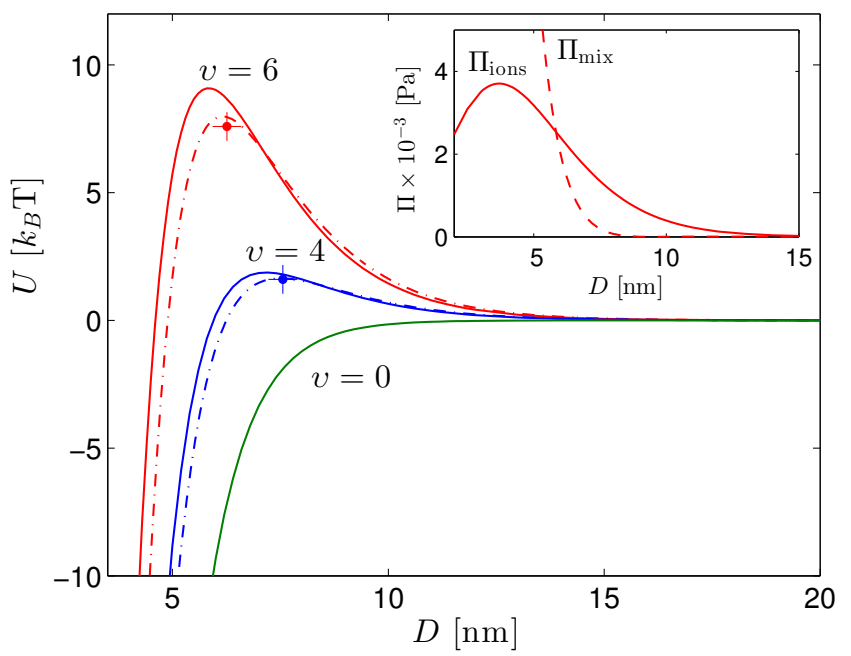

FIG. 2. (color online) The interaction potential $U(D)$ between electrically neutral and hydrophobic plates immersed in a mixture with $\phi_{0}, T$ and $n_{0}$ as in Fig. 11 When both ions are hydrophilic the interaction is purely attractive $\left(\Delta u^{ \pm}=6\right.$, green curve). A weak repulsive barrier $U_{\max }$ appears with antagonistic salts $\left(\Delta u^{+}=-\Delta u^{-}=4\right.$, blue curve), and its height increases with increasing $\Delta u^{+}-\Delta u^{-}\left(\Delta u^{+}=-\Delta u^{-}=\right.$ 6 , red curve). Full numerical solutions (solid curves) are in good agreement with linear theory (dash-dot curves), where we used $\kappa^{-1}=1.68 \mathrm{~nm}, \xi=0.94 \mathrm{~nm}$ and $l=1.78$. Two crosses are the approximation $\left(D_{\max }, U_{\max }\right)$ of Eq. (14). Inset: The two components of the osmotic pressure (see text) for the red curve. Here and in Fig. 3 the area of the hydrophobic plates is $S=0.01 \mu \mathrm{m}^{2}$ and $\Delta \gamma=0.2 / a^{2}$.

In order to better understand the physical origin of the repulsion we examine the components of the osmotic pressure. For symmetric plates, $\Pi$ can be recast in term of the mid-plane composition $\phi_{m}=\phi(z=0 ; D)$ and ion densities $n_{m}^{ \pm}=n^{ \pm}(z=0 ; D)$ as $\Pi(D)=\Pi_{\text {ions }}\left(n_{m}^{ \pm}\right)-$ $\Pi_{\text {mix }}\left(\phi_{m}\right)$, where

$$
\begin{aligned}
& \Pi_{\text {ions }}=k_{B} T\left(n_{m}^{+}+n_{m}^{-}-2 n_{0}\right), \\
& \Pi_{\text {mix }}=k_{B} T\left(f_{m}\left(\phi_{m}\right)-f_{m}\left(\phi_{0}\right)-\mu_{0}\left(\phi_{m}-\phi_{0}\right)\right) .
\end{aligned}
$$

The inset of Fig. 2 shows $\Pi_{\text {ions }}$ and $\Pi_{\text {mix }}$ for the $v=6$ potential curve. It is seen that $\Pi_{\text {ions }}$ is repulsive at $D \gtrsim$ $4 \mathrm{~nm}$; when the EDLs overlap this leads to an increase of $n_{m}^{ \pm}$. $\quad-\Pi_{\text {mix }}$ on the other hand is attractive. Since $U$ is the cumulative integral of $\Pi_{\text {ions }}-\Pi_{\text {mix }}$, a repulsive barrier is created by a range of $D$ for which $\Pi_{\text {ions }}>\Pi_{\text {mix }}$, corresponding to $D \gtrsim 6 \mathrm{~nm}$ in the Figure.

To better characterize the repulsive potential we solve the governing equations in the limit where the perturbations in the composition $\varphi=\phi-\phi_{0}$ and ion densities $\delta n^{ \pm}=n^{ \pm}-n_{0}$ are small [18]: $\delta n^{ \pm}=n_{0}\left(\Delta u^{ \pm} \varphi \mp \Psi\right)$, $\kappa^{-2} \nabla^{2} \Psi=\Psi-v \varphi, \kappa^{-2} \nabla^{2} \varphi=l^{2} \varphi+\omega^{2}(\Psi / v-\varphi)$, where $\kappa=\left(2 l_{B} n_{0}\right)^{1 / 2}$ is the Debye wavenumber and $\omega=$ $|v| /\left(l_{B} C\right)^{1 / 2}$ is a scaled $v$. Here, $l_{B}=e^{2} /\left(\varepsilon_{0} \varepsilon\left(\phi_{0}\right) k_{B} T\right)$ is the Bjerrum length at $\phi_{0}$ and $l=1 / \kappa \xi$ is the ratio of the Debye length and the modified correlation length $\xi: \xi=$ $(C / \tau)^{1 / 2}$, where $\tau=\partial^{2} f_{m}\left(\phi_{0}\right) / \partial \phi^{2}-n_{0}\left(\Delta u^{+}+\Delta u^{-}\right)^{2} / 2$.
The solution of the linear equations with the $z \rightarrow-z$ symmetry is:

$$
\begin{aligned}
& \Psi(z)=a_{1} \cosh \left(q_{1} z\right)-a_{2} \cosh \left(q_{2} z\right), \\
& \varphi(z)=b_{1} \cosh \left(q_{1} z\right)-b_{2} \cosh \left(q_{2} z\right) .
\end{aligned}
$$

The wavenumbers $q_{i}$ obey

$$
\left(q_{i} / \kappa\right)^{4}-\left(1+l^{2}-\omega^{2}\right)\left(q_{i} / \kappa\right)^{2}+l^{2}=0 .
$$

The amplitudes $a_{i}$ and $b_{i}$ are determined using the boundary conditions; in the special case where the plates are electrically neutral they are

$$
\begin{aligned}
a_{i} & =\frac{\Delta \gamma}{C} \frac{\kappa^{2} v}{q_{i}\left(q_{2}^{2}-q_{1}^{2}\right) \sinh \left(q_{i} D / 2\right)}, \\
b_{i} & =\frac{\Delta \gamma}{C} \frac{q_{i}^{2}-\kappa^{2}}{q_{i}\left(q_{2}^{2}-q_{1}^{2}\right) \sinh \left(q_{i} D / 2\right)}
\end{aligned}
$$

In the linear case it follows that

$$
\begin{aligned}
\frac{U}{k_{B} T}=S \times \frac{(\Delta \gamma)^{2}}{2 C} & {\left[\Lambda_{1} \frac{\operatorname{coth}\left(q_{1} D / 2\right)-1}{q_{1}}\right.} \\
& \left.-\Lambda_{2} \frac{\operatorname{coth}\left(q_{2} D / 2\right)-1}{q_{2}}\right],
\end{aligned}
$$

where

$$
\Lambda_{i}=\frac{q_{i}^{2}-\kappa^{2}}{q_{2}^{2}-q_{1}^{2}} .
$$

One can see from Eq. (11) that the interaction is $\propto(\Delta \gamma)^{2}$ and that the interplay between the two terms in brackets determines the nature of $U$. In the limit of vanishing solvation asymmetry $\omega \rightarrow 0$, we have from Eq. (8): $q_{1} \rightarrow \kappa$ and $q_{2} \rightarrow \xi^{-1}$, leading to $\Lambda_{1} \rightarrow 0$ and $\Lambda_{2} \rightarrow 1$. The resulting potential is attractive, as expected when ion solvation is absent due to critical adsorption [30].

For non-vanishing $\omega$, we focus on the region above $T_{c}$ for which $l>1+\omega$ and hence both $q_{1}$ and $q_{2}$ are positive real numbers. For sufficiently small $\omega$ we find

$$
q_{1} \cong \kappa \sqrt{1+\frac{\omega^{2}}{l^{2}-1}}, \quad q_{2} \cong \xi^{-1} \sqrt{1-\frac{\omega^{2}}{l^{2}-1}} .
$$

In this region, it is easy to show that $q_{2}>q_{1}>\kappa$ and thus $\Lambda_{1}>0$ and $\Lambda_{2}>0$. Therefore, the first term in brackets in Eq. (11) is repulsive while the second is attractive, leading to the existence of a maximum in the potential. The location and magnitude of this repulsive barrier are found by solving $\partial U / \partial D=0$. In the limit $D \gg q_{1}^{-1}, q_{2}^{-1}$ we find for $D_{\max }$ and $U_{\max }$ :

$$
\begin{aligned}
& D_{\max }=\frac{\log \left(\Lambda_{2} / \Lambda_{1}\right)}{q_{2}-q_{1}}, \\
& \frac{U_{\max }}{k_{B} T}=S \times \frac{(\Delta \gamma)^{2}}{C}\left[\frac{\Lambda_{1}}{q_{1}}\left(\frac{\Lambda_{2}}{\Lambda_{1}}\right)^{\frac{q_{1}}{q_{2}-q_{1}}}-\frac{\Lambda_{2}}{q_{2}}\left(\frac{\Lambda_{2}}{\Lambda_{1}}\right)^{\frac{q_{2}}{q_{2}-q_{1}}}\right] .
\end{aligned}
$$

Whether $U_{\max }$ is significant depends on the ratio of amplitudes $\Lambda_{2} / \Lambda_{1}$. 

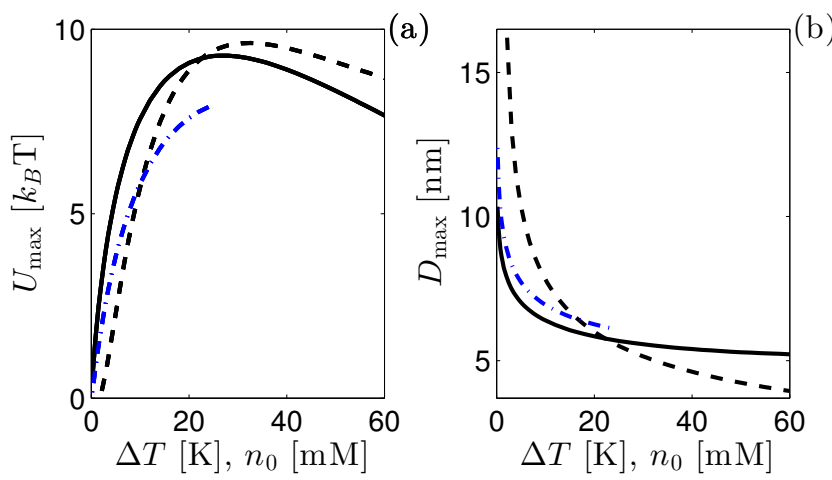

FIG. 3. (a) Barrier height and (b) location as a function of $\Delta T$ and $n_{0}$ for $v=6$ and $\Delta \gamma=0.2 / a^{2}$. Dashed curves are numerical results for $\phi_{0}=0.5, n_{0}=20 \mathrm{mM}$ and varying $\Delta T$; a repulsive barrier appears at $\Delta T \gtrsim 2 \mathrm{~K}$. For the solid curves $\phi_{0}=0.5, \Delta T=21.5 \mathrm{~K}$ and $n_{0}$ varies. Dash-dot curves are Eq. (14) for varying $n_{0}$ plotted in the validity range given by $l>1+\omega$.

At large enough colloid separations $D$ the repulsive tail of the interaction is $U / k_{B} T \simeq$ $S(\Delta \gamma)^{2} /\left(C / \Lambda_{1}\right) \exp \left(-q_{1} D\right) / q_{1}$. This expression is analogous to the regular Debye-Hückel result $U / k_{B} T \simeq S\left(\sigma^{2} / \varepsilon_{0} \varepsilon\right) \exp (-\kappa D) / \kappa$ for charged colloids [1]. In our theory $q_{1}$ is a modified Debye wavenumber, $C / \Lambda_{1}$ is a property of the medium, and $\Delta \gamma$ plays the role of an effective surface charge, reflecting the properties of the surface.

The comparison between the full potential and the analytical approximation Eq. (11) is shown in Fig. 2, For both $v=6(\omega=0.64)$ and $v=4(\omega=0.43)$, the linear theory (dash-dot curves) agrees quite well with the numerical solution (solid curves). Two circles in Fig. 2 show $D_{\max }$ and $U_{\max }$ evaluated using Eq. (14). Our analysis shows that $q_{1}$ and $\Lambda_{1}$ must be large enough for significant repulsion to appear. Hence, from Eq. (13) and Eq. (12) we conclude that $\omega$ should not be too small and $l$ not too large. The first requirement is satisfied by choosing antagonistic salts while the second dictates the temperature window given the salt concentration.

The two experimentally important quantities $U_{\max }$ and $D_{\max }$ are plotted in Fig. 3 (a) and (b), respectively. For increasing $\Delta T$ (dashed curves) a repulsive barrier first appears at $\Delta T \approx 2 \mathrm{~K}$, and it has a maximal value. The solid curves give results for varying $n_{0}$, showing again a maximum in $U_{\max }$. Dash-dot lines are $U_{\max }$ and $D_{\max }$ vs $n_{0}$ from Eq. (14) in the range $l>1+\omega$. Fig. 3 (a) shows that stabilization can be achieved far above the critical temperature. Thus, in principle the theory applies to experiments with completely miscible mixtures, e.g, water and alcohol, which in our theory is the $\chi \rightarrow 0$ limit of athermal mixtures.

The behavior of $U_{\max }$ is determined by the interplay between the attractive adsorption-related part of interaction and the repulsive solvation-related part. An increase in $\Delta T$ or $n_{0}$ increases $\xi^{-1}$ or $\kappa$, respectively. The result

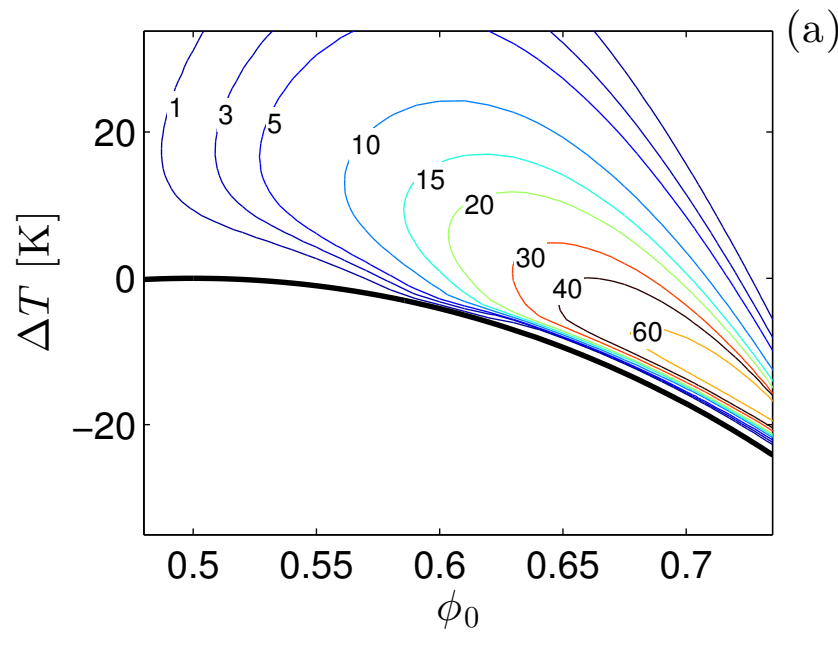

(a)

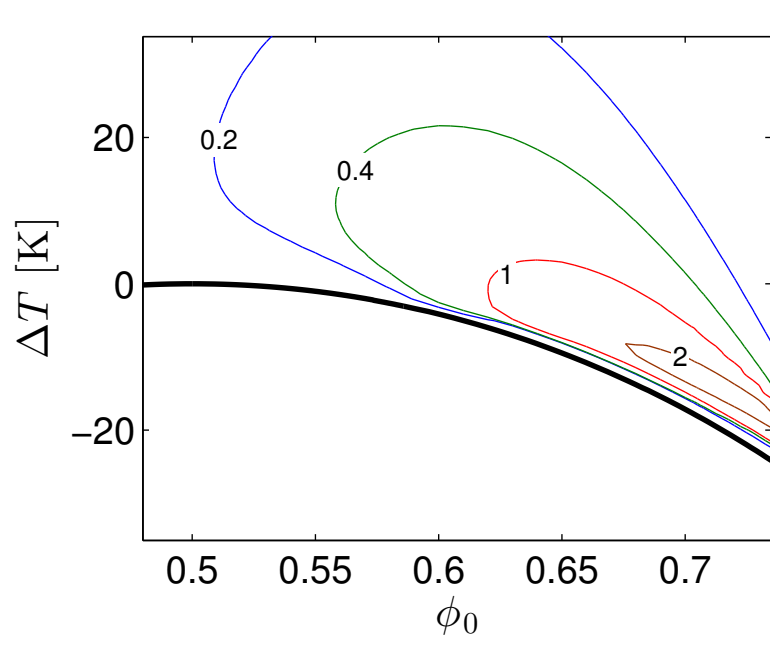

(b)

FIG. 4. The maximum of the interaction potential, $U_{T, \max }\left(\phi_{0}, T\right)$, in the $\phi_{0}-T$ plane including vdW attraction and using Derjaguin's approximation for a colloid radius $R=1 \mu \mathrm{m}$. (a) Contours of $U_{T, \max }$ for a Hamaker constant $A_{H}=0.2 \times 10^{-20} \mathrm{~J}$. (b ) The contour lines corresponding to $U_{T, \max }=3 k_{B} T$ for different Hamaker constants. The values of Hamaker's constant are indicated by the labels in units of $10^{-20} \mathrm{~J}$. Here $v=6, n_{0}=20 \mathrm{mM}$ and $\Delta \gamma=0.2 / a^{2}$.

in both cases is an increase in the wavenumbers $q_{i}$ and therefore a decrease in $D_{\max }$, see Fig. 3 (b). Furthermore, the relative magnitudes of the wavenumbers $q_{i}$ and the amplitudes $\Lambda_{i}$ change in a non trivial manner due to the coupling of the attractive and repulsive contributions, given by the parameter $l$ in the linear theory.

In the spirit of the DLVO theory, the more realistic case of spherical colloids of radius $R$ is evaluated by applying Derjaguin's approximation to the potential $U$ and adding the vdW interaction between the spheres: $U_{T}=\pi R \int_{D}^{\infty} U\left(D^{\prime}\right) \mathrm{d} D^{\prime}-A_{H} R /(12 D)$, where $A_{H}$ is the Hamaker constant. Contours of the maximum of $U_{T}$, $U_{T, \max }$, are shown in Fig. 4 (a) in the $\phi_{0}-T$ plane. Notice that $U_{T, \max }$ increases significantly for water-rich compositions $\left(\phi_{0}>0.5\right)$. The reason for this is twofold: (i) 


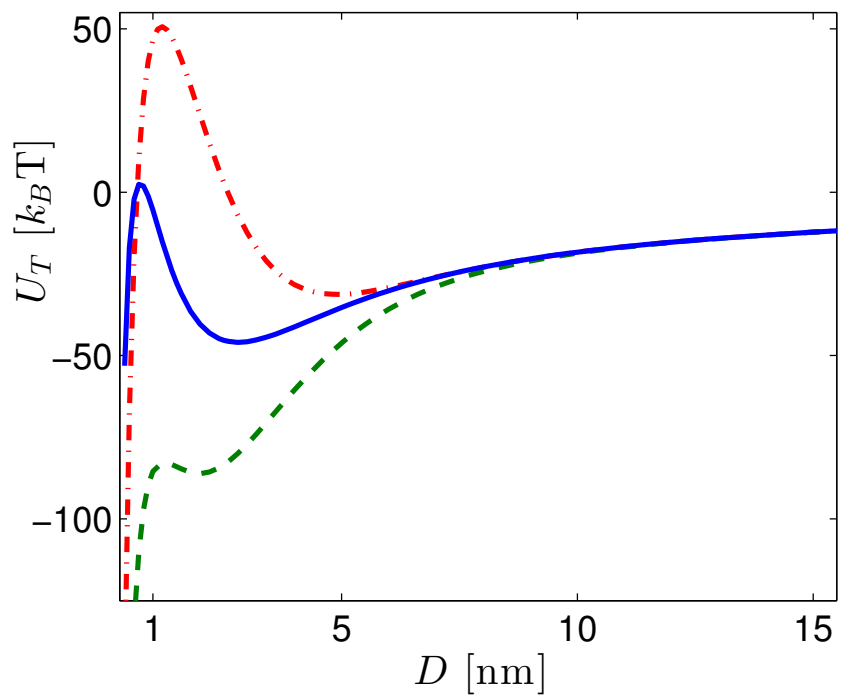

FIG. 5. $U_{T}(D)$ for two charged colloids with a surface charge density $\sigma=0.1 \mathrm{~nm}^{-2}$. When $\Delta \gamma=0$ and $\Delta u^{ \pm}=0$ (solid curve) the potential is marginally stable. For a hydrophobic surface, $\Delta \gamma=0.2 / a^{2}$, the potential is unstable for a hydrophilic salt $\left(\Delta u^{ \pm}=6\right.$, dashed curve) while it has a large barrier for an antagonistic salt $\left(\Delta u^{+}=-\Delta u^{-}=6\right.$, dash-dot curve). Here $n_{0}=20 \mathrm{mM}, \phi_{0}=0.5, \Delta T=21.5 \mathrm{~K}, R=1 \mu \mathrm{m}$ and $A_{H}=1 \times 10^{-20} \mathrm{~J}$.

the adsorption force is weaker at off-critical compositions and (ii) relative to the bulk composition, the water-poor layer on the surface is more attractive for hydrophobic ions. For hydrophilic colloids $(\Delta \gamma<0) U_{T, \max }$ would be larger at compositions $\phi_{0}<0.5$. While the absolute values of $U_{T, \max }$ do not depend on the sign of $\Delta \gamma$, the hydrophobicity or hydrophilicity of the colloids determines the ideal working region in the $\phi_{0}-T$ plane. The con-

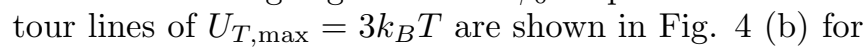
different values of the Hamaker constant. The area enclosed by this contour defines approximately the working conditions for a stable dispersion. Indeed, even for large values of the Hamaker constant a stable region exists for water-rich compositions and closer to the binodal curve. Hence, one could add only a small amount of co-solvent to an unstable dispersion of charge-free colloids to obtain a stable dispersion.

The linear solvation model adopted by us for its simplicity is a first-order approximation, and the study of more complex and realistic solvation models is an active area of research 22]. Nonetheless, the large repulsive barriers we predict are not restricted to a linear solvation model [31]. In addition, a significant barrier can be obtained also for weakly antagonistic salts $(v \simeq 1)$ if the plates are made more hydrophobic or hydrophilic (Eq.
(11)), or if the bulk composition is changed (Fig. 目(a)).

In Fig. 5 we show that addition of antagonistic salts can enhance the stability of charged colloids as well. When the colloids' surface and the ions are indifferent to the solvents, $\Delta \gamma=0$ and $\Delta u^{ \pm}=0$, as in the regular Poisson-Boltzmann theory, the effect of the mixture on the interaction is via the dependence of the dielectric constant on $\phi$. The result for $U_{T}(D)$, shown by the solid curve in Fig. [5 is a marginally stable potential. For hydrophobic colloids $(\Delta \gamma>0), U_{T}$ becomes attractive if the salt is hydrophilic (dashed curve), indicating a destabilization of the suspension. However, for hydrophobic colloids and an antagonistic salt (dash-dot curve) the repulsive barrier increases significantly, indicating a stabilization of the suspension. Here, the hydrophobic and positively charged colloids draw a larger amount of hydrophobic anions towards their surface, leading to an increase in the repulsive osmotic pressure of ions.

In conclusion, the theory predicts that significant potential barriers exist in a wide temperature and composition range and shows that neutral and charged colloids can be effectively suspended in a binary mixture by addition of antagonistic salts. It is worth noting that the specific adsorption of ions to the surface not discussed here will typically enhance the stabilization. The ion affinity to the wetting liquid will usually come hand in hand with a similar surface affinity thus enhancing the electrostatic repulsion.

The mechanism we describe is of potential use in numerous colloidal systems where currently only one solvent is employed and it is advantageous over existing methods in cases where the modification of the colloid surface chemistry is undesired. The most important requirement to achieve a stable suspension is to chose a salt in which the ions' solvation asymmetry in the mixture is large enough. We expect our results to be most beneficial for dispersing charge-free particles. For example, there have been large efforts recently in "transparent and conducting" electrodes for solar cell applications [32]. In these works graphite is typically sonicated to yield graphene sheets, and these sheets are dispersed using surfactants. Using evaporation or slow sedimentation these sheets assemble as a thin and conducting layer on top of a transparent substrate. In those works surfactants stay between graphene sheets and reduce the conductivity immensely. In this and other cases, using salts for the dispersion instead of surfactants could increase markedly the conductivity of the film.

Acknowledgments This work was supported by the Israel Science Foundation under grant No. 11/10, the COST European program MP1106 "Smart and green interfaces - from single bubbles and drops to industrial, environmental and biomedical applications", and the European Research Council "Starting Grant" No. 259205.
[1] W. B. Russel, D. A. Saville, and W. R. Schowalter. Colloidal Dispersions. Cambridge University Press, 1992.
[2] B. M. Law, J.-M. Petit, and D. Beysens. Adsorption- 
induced reversible colloidal aggregation. Phys. Rev. E, 57(5):5782-5794, 1998.

[3] Paul Hopkins, Andrew J. Archer, and Robert Evans. Solvent mediated interactions between model colloids and interfaces: A microscopic approach. J. Chem. Phys., 131(12):124704, 2009.

[4] D. Beysens and D. Estève. Adsorption phenomena at the surface of silica spheres in a binary liquid mixture. Phys. Rev. Lett., 54(19):2123-2126, May 1985.

[5] Jeroen S. van Duijneveldt and Daniel Beysens. Adsorption on colloids and flocculation: The influence of salt. J. Chem. Phys., 94(7):5222-5225, 1991.

[6] Mirjam E. Leunissen, Alfons van Blaaderen, Andrew D. Hollingsworth, Matthew T. Sullivan, and Paul M. Chaikin. Electrostatics at the oilwater interface, stability, and order in emulsions and colloids. Proc. Natl. Acad. Sci. U.S.A., 104(8):2585-2590, 2007.

[7] Mirjam E. Leunissen, Jos Zwanikken, Rene van Roij, Paul M. Chaikin, and Alfons van Blaaderen. Ion partitioning at the oil-water interface as a source of tunable electrostatic effects in emulsions with colloids. Phys. Chem. Chem. Phys., 9:6405-6414, 2007.

[8] Daniel Bonn, Jakub Otwinowski, Stefano Sacanna, Hua Guo, Gerard Wegdam, and Peter Schall. Direct observation of colloidal aggregation by critical casimir forces. Phys. Rev. Lett., 103(15):156101, Oct 2009.

[9] C. Hertlein, L. Helden, A. Gambassi, S. Dietrich, and C. Bechinger. Direct measurement of critical casimir forces. Nature, 451(7175):172-175, 2008.

[10] Ursula Nellen, Julian Dietrich, Laurent Helden, Shirish Chodankar, Kim Nygård, J. Friso van der Veen, and Clemens Bechinger. Salt-induced changes of colloidal interactions in critical mixtures. Soft Matter, 7:5360-5364, 2011.

[11] Van Duc Nguyen, Suzanne Faber, Zhibing Hu, Gerard H. Wegdam, and Peter Schall. Controlling colloidal phase transitions with critical casimir forces. Nat. Commun., 4:1584-, March 2013.

[12] Pradip B. Shelke, V. D. Nguyen, A. V. Limaye, and P. Schall. Controlling colloidal morphologies by critical casimir forces. Adv. Mater., 25(10):1499-1503, 2013.

[13] Akira Onuki and Hikaru Kitamura. Solvation effects in near-critical binary mixtures. J. Chem. Phys., 121(7):3143-3151, 2004.

[14] Yoav Tsori and Ludwik Leibler. Phase-separation in ioncontaining mixtures in electric fields. Proc. Nat. Acad. Sci., 104(18):7348-7350, 2007.

[15] Jos Zwanikken and René van Roij. Charged colloidal particles and small mobile ions near the oil-water interface: Destruction of colloidal double layer and ionic charge separation. Phys. Rev. Lett., 99:178301, Oct 2007.

[16] Jos Zwanikken, Joost de Graaf, Markus Bier, and René van Roij. Stability of additive-free water-in-oil emulsions. J. Phys.: Condens. Matter, 20(49):494238, 2008.

[17] Sela Samin and Yoav Tsori. Attraction between like- charge surfaces in polar mixtures. EPL, 95(3):36002, 2011.

[18] Ryuichi Okamoto and Akira Onuki. Charged colloids in an aqueous mixture with a salt. Phys. Rev. E, 84:051401, 2011.

[19] Akira Onuki and Ryuichi Okamoto. Selective solvation effects in phase separation in aqueous mixtures. Curr. Opin. Colloid Interface Sci., 16(6):525 - 533, 2011.

[20] Markus Bier, Andrea Gambassi, Martin Oettel, and Siegfried Dietrich. Electrostatic interactions in critical solvents. EPL, 95(6):60001, 2011.

[21] Sela Samin and Yoav Tsori. The interaction between colloids in polar mixtures above $t_{c}$. J. Chem. Phys., 136(15):154908, 2012.

[22] Markus Bier, Andrea Gambassi, and Siegfried Dietrich. Local theory for ions in binary liquid mixtures. J. Chem. Phys., 137(3):034504, 2012.

[23] C. Kalidas, Glenn Hefter, and Yizhak Marcus. Gibbs energies of transfer of cations from water to mixed aqueous organic solvents. Chem. Rev., 100(3):819-852, 2000.

[24] Yizhak Marcus. Gibbs energies of transfer of anions from water to mixed aqueous organic solvents. Chem. Rev., 107(9):3880-3897, 2007.

[25] Matej Kanduč, Ali Naji, Jan Forsman, and Rudolf Podgornik. Attraction between neutral dielectrics mediated by multivalent ions in an asymmetric ionic fluid. $J$. Chem. Phys., 137(17):174704, 2012.

[26] Jos W. Zwanikken and Monica Olvera de la Cruz. Tunable soft structure in charged fluids confined by dielectric interfaces. Proc. Nat. Acad. Sci., 110(14):5301-5308, 2013.

[27] Alexandre P. dos Santos and Yan Levin. Ion specificity and the theory of stability of colloidal suspensions. Phys. Rev. Lett., 106:167801, Apr 2011.

[28] T. Markovich, D. Andelman, and R. Podgornik. Surface tension of electrolyte solutions: A self-consistent theory. ArXiv e-prints, page 1305.3142, 2013.

[29] Samuel Safran. Statistical Thermodynamics of Surfaces, Interfaces, and Membranes. Westview Press, New York, 1994.

[30] Michael E. Fisher and Pierre-Gilles de Gennes. Phénomènes aux parois dans un mlange binaire critique. C. R. Seances Acad. Sci., Ser. B, 287:207, 1978.

[31] We verified that our conclusions are not restricted to the choice of a bilinear solvation energy. Using data from Refs. 23, 24] we have fitted, using polynomials, the experimental solvation energy for antagonistic salts. For this more complex model, our results remain qualitatively the same and quantitatively similar.

[32] Matat Buzaglo, Michael Shtein, Sivan Kober, Robert Lovrincic, Ayelet Vilan, and Oren Regev. Critical parameters in exfoliating graphite into graphene. Phys. Chem. Chem. Phys., 15:4428, 2013. 